\title{
Endovascular Management of Direct Carotid- Cavernous Fistula: Evolution of Cost Effective Sandwich Technique
}

\author{
Surya N. Prasad ${ }^{1}$ Vivek Singh ${ }^{1} \quad$ Deb K. Boruah² \\ ${ }^{1}$ Department of Radiodiagnosis, Sanjay Gandhi Postgraduate \\ Institute of Medical Sciences, Lucknow, Uttar Pradesh, India \\ 2Department of Radiodiagnosis, Tezpur Medical College and \\ Hospital, Tezpur, Assam, India \\ ${ }^{3}$ Department of Radiodiagnosis, Apollo hospital, Lucknow, \\ Uttar Pradesh, India \\ ${ }^{4}$ Department of Ophthalmology, Sanjay Gandhi Postgraduate \\ Institute of Medical Sciences, Lucknow, Uttar Pradesh, India
}

Rajendra V. Phadke ${ }^{3}$

Kumudini Sharma ${ }^{4}$

Vikas Kannaujia ${ }^{4}$

\begin{abstract}
Address for correspondence Vivek Singh, DNB, Department of Radiodiagnosis, Sanjay Gandhi Post Graduate Institute of Medical Sciences, Rae Bareilly Road, Lucknow, Uttar Pradesh 226014, India (e-mail: singhvivek79@rediffmail.com).
\end{abstract}

\begin{abstract}
Keywords

- carotid cavernous fistula

- embolization

- parent vessel occlusion

- detachable balloons

- sandwich technique

Objective There is a direct fistulous connection between the cavernous segment of the internal carotid artery and cavernous sinus in cases of direct carotid-cavernous fistula (CCF). Endovascular embolization is the mainstay of management in this condition. This study is about the evolution of endovascular treatment methods and the development of a cost-effective technique for embolization of direct CCF at a tertiary care center.

Materials and Methods A retrospective analysis was performed of all the cases of direct type CCF embolized by endovascular techniques in our department from 2008 to 2018.Clinical follow-up of these patients was done at 1 week, 3 months, and 6 months.

Results A total of 45 patients with 40 having a prior history of head trauma were included in this study. All cases were treated with a transarterial route except one which was treated with the transvenous approach. Detachable balloons were used in 12 (26.67\%) patients, only detachable coils in 14 cases (31.11\%), both detachable balloons and coils in 9 cases (20\%), and both detachable and push coils, that is, sandwich technique in 8 cases (17.78\%). Parent arterial occlusion was performed in 10 patients (22.22\%). There was complete resolution of chemosis and bruit in all the patients.

Conclusion Endovascular treatment is the mainstay of management in direct CCF. Using more fibered thrombogenic coils in a sandwich manner decreases the cost of the treatment significantly.
\end{abstract}

\section{Introduction}

Carotid-cavernous fistulas (CCFs) are abnormal fistulous connections between the cavernous sinus and carotid circulation. When there is a direct fistulous connection between the cavernous sinus and cavernous segment of the internal carotid artery (ICA), it is called direct or type-A CCF. ${ }^{1}$ Generally, these patients have a prior history of head injury and then in due course of time, there is the subsequent development of chemosis, proptosis, bruit, headache, and/or gradual decrease in vision either unilaterally or bilaterally.

Since direct (type A) CCFs are high flow fistulas with acute/ subacute presentation and may cause serious complications, such as permanent vision loss or intracranial hemorrhage, they have to be treated early. They nearly always need intervention, either endovascular or surgical. Surgery as of now in these cases is considered as last resort and has high chances of complications; however, it may be needed in cases 
where endovascular treatment has failed or not possible. Sometimes surgery can complement endovascular treatment by helping in exposing superior ophthalmic vein in cases where there is no other suitable arterial or venous access is available. Endovascular embolization of CCF is preferred as the first line of treatment. ${ }^{2}$ Endovascular management has evolved from the use of detachable balloons to coils and other embolic agents. Here, we present our experience in endovascular management and our endeavor to decrease the cost of the treatment of direct CCF at our institution.

\section{Materials and Methods}

A retrospective analysis was done of all the cases of direct CCF that were managed by endovascular means in our department from 2008 to 2018. Clinical signs and symptoms, history of injury, duration of symptoms, and demographic details were recorded at the time of presentation. Endovascular embolization of the fistulae was performed using detachable balloons and/or detachable/push coils depending on availability, patient's affordability, type of fistula, and ease of use. Compliant balloon like Hyperglide $4 \mathrm{~mm} \times 20 \mathrm{~mm}(\mathrm{eV} 3$, Irvine, California, United States) or Scepter C $4 \mathrm{~mm} \times 20 \mathrm{~mm}$ (Microvention, Aliso Viejo, California, United States) were kept in ICA across the defect in few cases for balloon assisted coiling. Postprocedure, the patient was followed-up at 1 week, 3 months, and 6 months clinically by an ophthalmologist for improvement of symptoms.

\section{Results}

A total of 45 cases (30 males and 15 females, 2:1 ratio) were included in the study. Among them, 40 patients (88.89\%) had a previous history of significant head injury. Three patients had rupture of preexisting ICA aneurysm into the cavernous sinus, whereas another two patients suspected to have connective tissue disorder (CTD) presented with spontaneous direct CCF.

The average ages at presentation for direct CCF were 27.47 and 37.13 years for males and females, respectively. Patients presented clinically and came for treatment from as early as 15 days after initial insult to as late as 24 months after the injury with an average duration of symptoms at a presentation being 7.5 months. In 29 cases (64.44\%), right cavernous sinus was involved and left cavernous sinus was primarily affected in 16 cases (35.56\%). All the cases were treated via transarterial approach except one where the cavernous sinus was coiled transvenously, as transarterial embolization (TAE) could not be done due to very tortuous and difficult anatomy. In another case, spontaneous closure of the fistula occurred with manual compression of neck vessels, while the patient was in waiting for endovascular embolization. In one case, the site of ICA defect into cavernous sinus could not be cannulated and the procedure was abandoned. After carefully reviewing digital subtraction angiography (DSA) images retrospectively in that case, actually we found that the CCF defect was slit like with superimposed intimal flaps behaving as a valve and preventing the microcatheter to pass into the cavernous sinus. We advised the patient for manual compression therapy. On follow-up, there occurred gradual improvement in clinical symptoms and the patient did not agree for a repeat attempt of endovascular embolization. We did embolization with only detachable balloons in $12(26.67 \%)$ patients, only detachable coils in 14 cases (31.11\%), used both detachable balloons and coils in 9 cases (20\%), and both detachable and push coils in 8 cases (17.78\%). Parent arterial occlusion was performed in 10 patients (22.22\%). On postprocedure follow-up at 6 months, there was complete resolution of bruit, proptosis, and chemosis in all treated patients. Four patients, who had complete vision loss at presentation, did not show improvement in vision. The other four patients also showed features of persistent sixth cranial nerve palsy.

\section{Discussion}

CCFs are the abnormal connection between the cavernous sinus and carotid circulation. Based on angioarchitecture, they were classified as direct (type A) and indirect (types B, C, and D) types by Barrow et al. ${ }^{1}$

When there is a defect in the ICA wall resulting in its direct communication with cavernous sinus, it is called direct or type-A CCF. In the case of indirect CCF, the fistulous communication is between the cavernous sinus and dural branches of the carotid artery (either internal or external carotid artery [ECA] or both).

Indirect CCF are further subdivided as follows:

- Type B: fistulous connection between the cavernous sinus and the dural branches of ICA.

- Type C: fistulous connection between the cavernous sinus and the dural branches of ECA.

- Type D: fistulous connection between the cavernous sinus and the dural branches of both ICA and ECA.

Direct CCFs are high-flow fistulas, mostly posttraumatic ( $85 \%$ cases), and usually result from head trauma (with or without skull base fracture). Overall, 0.2 to $0.3 \%$ of cases of craniofacial trauma present as direct CCF clinically. ${ }^{2-6}$ In our study, a history of significant head injury (road traffic accidents, penetrating trauma from gun-shot injury, and fall from height) was present in the majority ( $89 \%)$ of cases. Demographically the most vulnerable group is young male adults and this fact is also reflected in our study with a lower mean age (27.17 years) for male patients. ${ }^{3}$ This pattern is attributed to the assumption that the risk-taking behavior of male young adults in society makes them prone to injuries including head trauma.

In a minority ( $15 \%)$ of cases, direct CCF may result from spontaneous rupture of preexisting cavernous ICA aneurysm into the cavernous sinus. Rarely, direct CCF may occur in association with CTD like Marfan's syndrome, Ehlers-Danlos syndrome, pseudoxanthoma elasticum, fibromuscular dysplasia, and osteogenesis imperfecta. ${ }^{2-5}$ In Ehlers-Danlos syndrome, intracranial arteries have type-3 collagen defects and decreased elastic tissue in the vessel wall. ${ }^{6}$ In these cases, the inherent weakness of arterial wall media makes them prone to develop 
CCF spontaneously or after trivial trauma. This also creates a challenge for the interventionist to treat these patients, as they are more prone to develop intra- or postprocedure complications in the form of puncture site hematoma, arterial dissection, pseudoaneurysm formation, and even arterial perforation during the procedure. Two of our cases who developed spontaneous direct CCF were suspected to have CTD clinically; however, we could not find any conclusive evidence of the disease. Due care was exercised while handling the catheters, wires, contrast injection, and coiling during endovascular embolization of these cases and the procedures remained uneventful.

Clinical features are similar in both types (traumatic or spontaneous) of direct CCF. The classic presentation is described as Dandy's triad consisting of exophthalmos, bruit, and conjunctival chemosis. ${ }^{2-4,7,8}$ The predominant venous drainage pathway determines how the patient will present clinically. ${ }^{1,4,9,10}$ The venous reflux may occur into superior/ inferior ophthalmic veins (resulting in the development of proptosis, chemosis, orbital bruit, eye congestion, and exposure keratitis), cortical veins (resulting in headache and venous infarcts), pterygoid venous plexus and petrosal sinuses (resulting in headache and tinnitus), or medially to opposite cavernous sinus through intercommunicating veins (resulting in bilateral ocular signs and symptoms).

In anteriorly draining fistulas, a persistent increase in orbital venous pressure causes dilatation of superior/inferior ophthalmic veins, retroorbital soft-tissue edema, and increased intraocular pressure (IOP) resulting in compromised retinal perfusion and decreased visual acuity. ${ }^{2-4}$ Subsequently in longstanding cases optic nerve atrophy may ensue with total vision loss which is one of the most feared complications of direct $\mathrm{CCF}^{2}$ In our study, four patients had a complete loss of vision on the affected side at the presentation which did not improve post embolization. All of them had clinical symptoms for a long duration ( 24 months) and they sought medical help very late in the course of the disease. Thus, delay in diagnosis and management had adversely affected overall visual prognosis in these cases.

In predominantly posteriorly draining fistulas, patients may not have ophthalmic symptoms and sometimes have complaints of only troublesome bruit or tinnitus. This constitutes "white-eye syndrome" and potentially dangerous in the sense that the patient is not aware of the disease resulting in a delay in diagnosis/treatment and putting him on the risk of developing other more serious complications like venous infarct/intracranial hemorrhage or brainstem edema/bleed., ${ }^{2,511}$ One case in our study who presented with mild chemosis, proptosis, facial numbness, and hemiparesis, we noticed significant brainstem edema on magnetic resonance imaging (MRI) images. On DSA, the CCF had prominent venous drainage posteriorly into inferior petrosal sinus with congestion of basal veins of Rosenthal. Postembolization, the patient was cured of his clinical symptoms and follow-up MRI showed resolution of the brainstem edema.

Radiological diagnosis can be made on computed tomography (CT) or MRI; however, DSA is the gold standard for diagnosis of $\mathrm{CCF}^{2-4,7}$ DSA accurately depicts the site of fistula, predominant venous drainage pattern, presence of steal phenomenon, and vascular anatomy. An attempt has been made by Chi et al in grading direct CCF into small, medium, and large sizes depending on the opacification of intracranial arteries during DSA. ${ }^{10}$ This grading provides insight into the degree of shunting across fistula and may help in preplanning the procedure. In small- and mediumsized CCF, selective embolization of the fistula is advised, whereas in large size CCF, trapping of the fistula with ICA occlusion may be needed. In all our cases where parent vessel occlusion was done, the fistula was large.

Several maneuvers are described in the literature for localizing the exact site of the fistula. In Heuber's maneuver, vertebral artery injection is performed after compressing the common carotid artery (CCA) on the same side and the fistula fills through the patent posterior communicating artery. ${ }^{12}$ Alternatively in the Mehringer-Hieshima maneuver, the tip of the diagnostic catheter is kept in ipsilateral ICA and then manual compression is applied over the CCA on the same side while taking acquisition at a slower frame rate. This results in slow opacification of CCF and better localization of the fistula site. ${ }^{2}$

The treatment of direct CCF has evolved. Historically ligation of CCA/ICA or trapping of the fistula was done surgically but carried high morbidity and mortality. Now surgery has only a complementary role like exposing ophthalmic vein in cases where appropriate vascular access is not available. ${ }^{2-5,7}$ First-line treatment is endovascular embolization. In cases of direct $\mathrm{CCF}$, embolization via arterial route is preferred with the preservation of ICA patency wherever possible., ${ }^{2,3,8,9,13}$ Detachable silicone balloons were used frequently as an inexpensive embolizing agent for direct CCF with excellent success rates in various series., ${ }^{2,7-9,13}$ In our study, 12 (26.6\%) cases of direct CCF were treated with detachable balloons only (-Fig. 1) and in 9 (20\%) cases, we used both detachable balloons and coils. In the procedure, the detachable balloon mounted on a microcatheter is partially inflated to help it flow directed into the cavernous sinus through the fistula. Then the balloon is fully inflated to attain size greater than fistula defect to seal the same before detachment. It worked as an excellent and inexpensive embolizing material in cases where the placement of the balloon was easy. However, we did experience certain problems with the use of a balloon. One may need more than one balloon depending on the size, morphology, and number of CCF defects. The treating interventionist has to be careful about osmolality of the contrast used, as the balloon may rupture in cases of hyperosmolar contrast or may deflate if a hypoosmolar contrast agent is used for inflation. During the procedure, repeated inflation-deflation of the balloon within cavernous sinus should be avoided as this increases the chances of thromboembolic cerebrovascular events. ${ }^{7,8,9}$ Also the patient needs to keep his neck in certain partially extended posture for a day or two and avoid violent movement or straining activities like vigorous coughing, sneezing, or repeated yawning as the balloon may displace/dislodge from the region of cavernous sinus defect. Sometimes in acute cases, the balloon may get perforated by fractured bony fragment in the region of the cavernous 


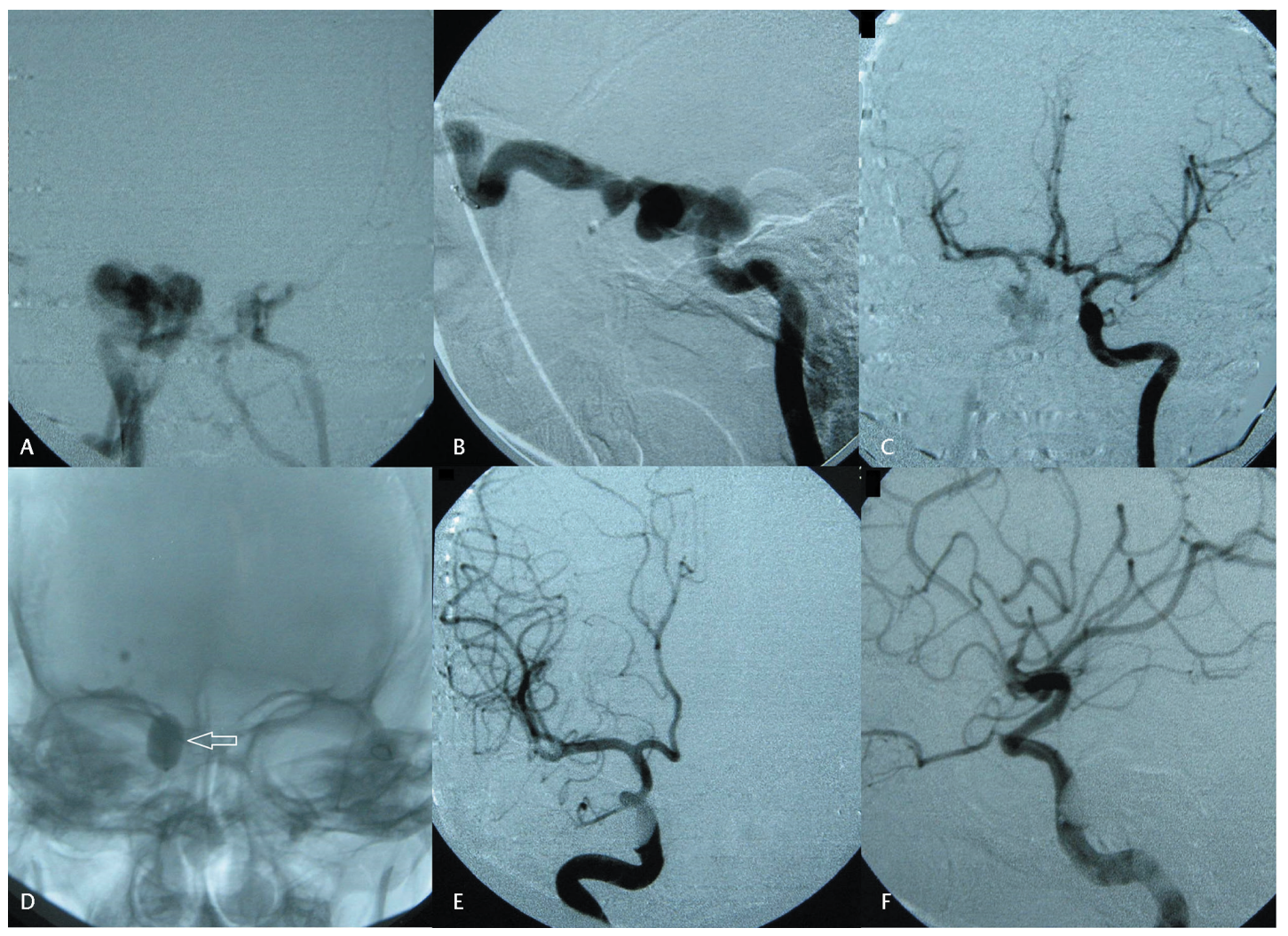

Fig. 1 (A) Anteroposterior (AP) and (B) lateral projections of right internal carotid artery (ICA) digital subtraction angiography (DSA) showing direct carotid-cavernous fistula (CCF) with early opacification of cavernous sinus and superior ophthalmic vein. Also noticed complete steal phenomenon with nonopacification of distal ICA and its branches. (C) Left ICA DSA showing good cross flow. (D) Detachable balloon (arrow) deployed at the site of CCF defect. (E) AP and (F) lateral postprocedure projections of right ICA DSA showing complete occlusion of CCF defect and nonopacification of cavernous sinus in arterial phase.

sinus. ${ }^{2,7}$ In cases of small defect or tortuous anatomy, sometimes it is not possible to negotiate the catheter into the cavernous sinus through the fistula for placement of the balloon. Also, the rate of reintervention/recurrence is more in balloon embolization as the packing of the fistula defect is not always optimum. ${ }^{7,13,14}$ Too large defect size, very tortuous and unfavorable anatomy, placement of multiple balloons, and incomplete embolization in the first session were considered important risk factors for failed treatment/recurrence. ${ }^{2,7,12,13}$ We needed more than one treatment session in 8 out of 21 cases $(38.09 \%)$ where detachable balloons were used as primary embolizing material. In nine cases (42.86\%), we had to use detachable coils to pack the cavernous sinus along with balloon embolization. Also, conversion of the procedure to parent artery occlusion was more in patients where we used detachable balloons as the primary embolizing material, as the behavior of balloons were unpredictable in several cases. ${ }^{23,6}$ In 8 out of 21 cases (38.09\%) where we used a detachable balloon as primary embolizing material, finally we had to go for parent artery occlusion to completely treat residual disease. Our results with detachable balloons were not as great as reported in the literature. ${ }^{7}$ After 2010 to 2011, the detachable balloons were phased out of the market and became increasingly unavailable, so we completely switched over to detachable coils for direct CCF embolization.

In $14(31.11 \%)$ cases, we performed embolization using detachable coils only ( - Fig. 2). They were easier to deploy with accurate placement and good packing of the fistula, as well as cavernous sinus, however, with a significant increase in the overall cost of the procedure. In developing countries, like one of ours, the cost of the procedure is a very important factor in the choice of therapy for the patients. So, in due course and with more experience, we devised a technique (may be referred to as "sandwich technique") to decrease the overall cost of treatment. After selectively cannulating the CCF defect with large size microcatheter ( 0.021 compatible), we start coiling with three-dimensional (3D) detachable coils for precise placement and to build a framework. Thereafter we use several fibered platinum push coils for the adequate packing of the cavernous sinus. These fibered coils increase thrombogenicity of the coil mass and thereby favoring early occlusion of the fistula site. During final coiling, while reaching near the ICA defect, we again use detachable coils to adequately pack and control the placement of coils so that inadvertent prolapse of the coil loop in the ICA can be avoided. Alternatively, an intra-arterial compliant balloon may be inflated near the ICA defect to preserve ICA patency during the procedure. ${ }^{15}$ In this way, the fibered push coils are "sandwiched" between detachable coils, and we performed endovascular embolization with "sandwich technique" in eight cases (17.78\%). Simultaneous use of compliant intra-arterial balloon which was kept in ICA across the defect was done in seven cases during coiling of the fistula. Keeping the compliant balloon in ICA during coiling guides, the interventionist about the patency of the parent vessel. All cases done with sandwich technique showed complete obliteration 


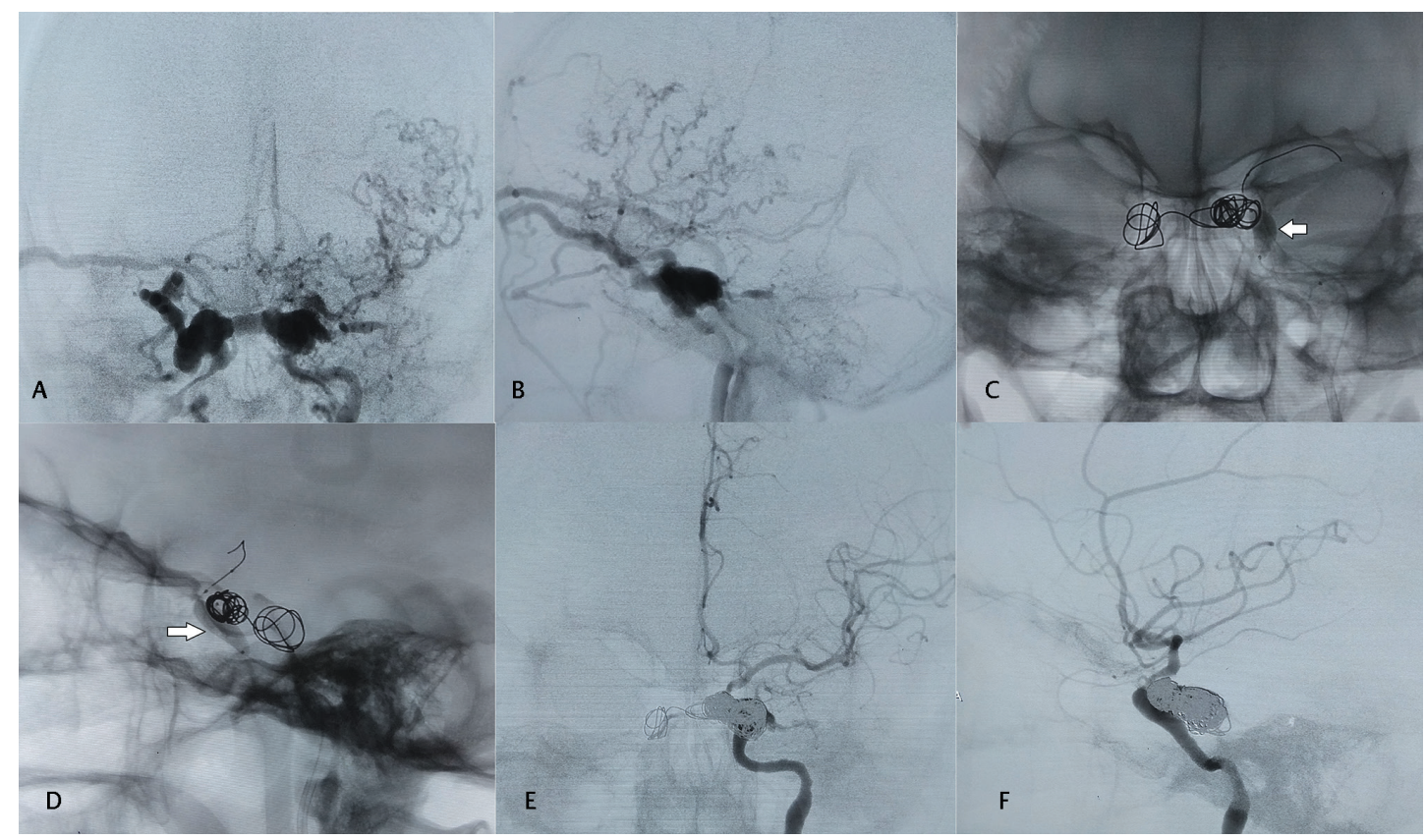

Fig. 2 (A) Anteroposterior (AP) and (B) lateral projections of left internal carotid artery (ICA) digital subtraction angiography (DSA) showing direct carotid-cavernous fistula (CCF) with opacification of bilateral cavernous sinuses, right superior ophthalmic vein, near complete steal phenomenon and contrast reflux into cortical veins on left side. Left superior ophthalmic vein was thrombosed and the patient presented with ocular symptoms predominantly on right side. (C) AP and (D) lateral projections showing a compliant balloon (arrow) in left cavernous ICA inflated at the site of CCF defect and initial deployment of coils in cavernous sinus and intercommunicating venous channel. (E) AP and (F) lateral projections of left ICA DSA showing cavernous sinus packed with multiple coils, occlusion of CCF and patent left ICA with good distal branches opacification..

of CCF on postprocedure DSA and clinical improvement on follow-up. We did not find any other study in the literature demonstrating the use of fibered platinum push coils in direct CCF embolization, which helped a lot in decreasing the overall cost of the procedure without compromising both the early and long-term outcome.

Parent artery occlusion was performed in 10 (22.22\%) patients (-Fig. 3). In cases where it is not possible to preserve ICA like severely damaged/dysplastic vessel wall, extensive tortuosity or active ongoing bleeding, one has to go for parent artery occlusion. ${ }^{2}$ Also in cases of failed surgical intervention, this may remain the only option left. In cases where there is inadequate or incomplete closure of CCF defect after embolization, one may need to perform parent artery occlusion to stop filling of the fistula after ensuring good cross-flow on balloon occlusion test. In our experience, we had to convert approximately $38 \%$ of the embolization procedure into parent artery occlusion where we used detachable balloons as primary embolizing material. This high rate of conversion into parent artery occlusion is attributed to the propensity of the detachable balloons to behave unpredictably in several cases leading to inadequate packing of CCF defect.

So far technique of the procedure (parent artery occlusion) is concerned, the principle is to trap the fistula site cutting-off both antegrade and retrograde blood supply to CCF. Alternatively, one can start with coiling the cavernous sinus itself and gradually coming back into ICA lumen covering the fistula site and proximal adjacent ICA segment. Before proceeding for parent artery occlusion, it is very important to ensure that the patient would tolerate it without producing neurological deficits. This is done by balloon occlusion test and demonstrating good cross-flow through the circle of Willis. ${ }^{2,16}$ In chronic cases, the ICA becomes significantly tortuous in course. In many of these high-flow fistulas, most of the flowing ICA blood passes into the venous system through the fistulous communication producing steal phenomenon and relative hypoperfusion of distal circulation. This, in turn, stimulates the development of collaterals and good crossflow through the circle of Willis. If the patient is tolerating near-complete steal phenomenon without any neurological deficit, in all probability, he/she will also tolerate parent artery occlusion and in these cases, even preprocedure balloon occlusion test is not necessary. ${ }^{2}$ However, in cases where there is no significant steal, one must perform a balloon occlusion test and demonstrate good cross-flow before going for parent artery occlusion. Six patients in our study had the presence of complete steal at the time of diagnostic DSA. The rest tolerated balloon occlusion test fairly well. None of our cases developed significant neurological deficit post parent vessel occlusion.

In literature, several cases are described where glue, onyx, or polyvinyl alcohol (PVA) particles were used by the interventionist in combination with detachable coils to adequately pack the fistula with variable results. ${ }^{17,18}$ Using liquid/particle embolizing agents for these high-flow conditions carry a high-complication rate like nontarget embolization of cortical veins or an arterial embolic stroke. ${ }^{17,18}$ We did not use liquid or particle agents for direct CCF embolization in any of our cases. 


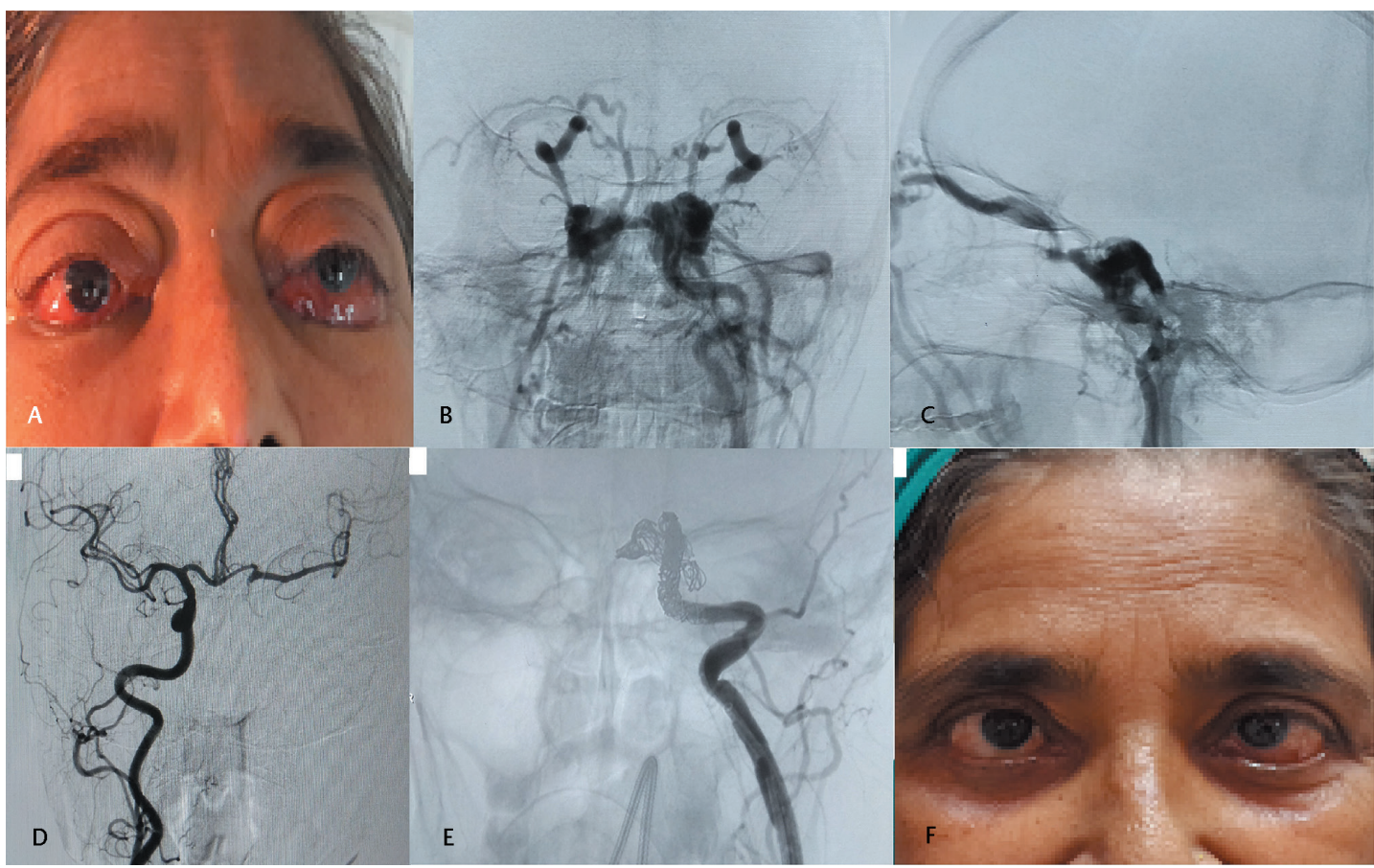

Fig. 3 (A) Clinical image at presentation showing proptosis and severe chemosis bilaterally. (B) Anteroposterior (AP) and (C) lateral projections of left left internal carotid artery (ICA) digital subtraction angiography (DSA) showing direct carotid-cavernous fistula (CCF) with early opacification of bilateral cavernous sinuses and superior ophthalmic veins. Complete steal is present with nonopacification of distal branches. (D) Right ICA DSA shows good cross flow through patent anterior communicating artery. (E) Parent artery occlusion with multiple coils. (F) Postprocedure day 3 clinical image showing complete resolution of proptosis and chemosis with features of residual sixth cranial nerve palsy on left side

Sometimes, there is incomplete occlusion of fistula after transarterial embolization. In those cases, subsequent transvenous (mostly through inferior petrosal sinus) approach may be needed to coil and occlude the fistula. At times simultaneous arterial and venous routes are used when through one route complete occlusion is not possible. ${ }^{19}$

Another endovascular treatment option is the placement of stent graft in cavernous ICA covering the fistula defect with or without coiling of the cavernous sinus. ${ }^{20}$ This is especially of great value in cases where the patient does not tolerate the balloon occlusion test. Stent graft placement ensures patency of the native artery, as well as sealing of the fistula site. In chronic cases or cases with delayed presentation and unfavorable anatomy, it is very difficult to navigate through and place the graft in cavernous ICA with good vessel wall opposition. This is due to the limited longitudinal flexibility of available stent grafts. At present, there is limited availability of compatible devices in the market and lack of long-term safety data in the literature regarding the use of stent graft for CCF treatment. Stent graft complications include endoleak, vasospasm, coverage of perforators, dissection, thrombosis, distal thromboembolism, and even perforation. Moreover, patient has to take antiplatelet agents subsequently to prevent intrastent-graft thrombosis in the future. ${ }^{20}$ There are studies where flow-diverters have been used for CCF treatment but again they are expensive and immediate occlusion of fistula does not occur and also carries a high-repeat procedure rate. ${ }^{21}$ In the future, more flexible cheap stent grafts or something like covered or coated flow-diverters may be the ideal device for endovascular management of direct CCF.

\section{Conclusion}

Direct CCFs are high-flow fistulas and mostly posttraumatic endovascular treatment is the mainstay of management. With the use of fibered platinum push coils in the above described "sandwich technique," we were able to reduce the expenses significantly without compromising the outcome. The future lies in the development of cost-effective flexible and more negotiable covered flow diverter like devices for CCF management.

\section{Conflict of Interest}

None declared.

\section{References}

1 Barrow DL, Spector RH, Braun IF, Landman JA, Tindall SC, Tindall GT. Classification and treatment of spontaneous carotid-cavernous sinus fistulas. J Neurosurg 1985;62(2):248-256

2 Korkmazer B, Kocak B, Tureci E, Islak C, Kocer N, Kizilkilic O. Endovascular treatment of carotid cavernous sinus fistula: a systematic review. World J Radiol 2013;5(4):143-155

3 Joshi DK, Singh DD, Garg DD, Singh DH, Tandon DM. Assessment of clinical improvement in patients undergoing endovascular coiling in traumatic carotid cavernous fistulas. Clin Neurol Neurosurg 2016;149:46-54 
4 Henderson AD, Miller NR. Carotid-cavernous fistula: current concepts in aetiology, investigation, and management. Eye (Lond) 2018;32(2):164-172

5 Williams ZR. Carotid-cavernous fistulae: a review of clinical presentation, therapeutic options, and visual prognosis. Int Ophthalmol Clin 2018;58(2):271-294

6 Adham S, Trystram D, Albuisson J, et al. Pathophysiology of carotid-cavernous fistulas in vascular Ehlers-Danlos syndrome: a retrospective cohort and comprehensive review. Orphanet J Rare Dis 2018;13(1):100

7 Gao BL, Wang ZL, Li TX, Xu B. Recurrence risk factors in detachable balloon embolization of traumatic direct carotid cavernous fistulas in 188 patients. J Neurointerv Surg 2018;10(7):704-707

8 Phadke RV, Kumar S, Sawlani V, Mazumdar B, Gujral RB. Traumatic carotid cavernous fistula: anatomical variations and their treatment by detachable balloons. Australas Radiol 1998;42(1):1-5

9 Holland LJ, Mitchell Ranzcr K, Harrison JD, Brauchli D, Wong Y, Sullivan TJ. Endovascular treatment of carotid-cavernous sinus fistulas: ophthalmic and visual outcomes. Orbit 2019;38(4):290-299

10 Chi CT, Nguyen D, Duc VT, Chau HH, Son VT. Direct traumatic carotid cavernous fistula: angiographic classification and treatment strategies. Study of 172 cases. Interv Neuroradiol 2014;20(4):461-475

11 Chan FH, Shen CY, Liu JT, Li CS. Brainstem hemorrhage caused by direct carotid-cavernous fistula. A case report and literature review. Interv Neuroradiol 2014;20(4):487-494

12 Huber P. A technical contribution of the exact angiographic localization of carotid cavernous fistulas. Neuroradiology 1976;10(5):239-241

13 Stéphan S, Blanc R, Zmuda M, et al. Endovascular treatment of carotid-cavernous fistulae: Long-term efficacy and prognostic factors. J Fr Ophtalmol 2016;39(1):74-81
14 Lin TC, Mao SH, Chen $\mathrm{CH}$, et al. Systematic analysis of the risk factors affecting the recurrence of traumatic carotid-cavernous sinus fistula. World Neurosurg 2016;90:539-545.e1

15 Andrade G, Ponte De Souza ML, Marques R, Silva JL, Abath C, Azevedo-Filho HR. Endovascular treatment of traumatic carotid cavernous fistula with balloon-assisted sinus coiling. A technical description and initial results. Interv Neuroradiol 2013;19(4):445-454

16 Hassan T, Rashad S, Aziz W, Sultan A, Ibrahim T. Endovascular modalities for the treatment of cavernous sinus arteriovenous fistulas: a single-center experience. J Stroke Cerebrovasc Dis 2015;24(12):2824-2838

17 Ohlsson M, Consoli A, Rodesch G. Endovascular treatment of carotico-cavernous fistulas with acrylic glue: a series of nine cases. Neuroradiology 2016;58(12):1181-1188

18 He X, Duan C, Lai L, et al. Comparison of polyvinyl alcohol copolymer with detachable balloons for the embolisation of direct carotid cavernous fistula: a single-centre experience. Eur Radiol 2017;27(11):4730-4736

19 Du B, Zhang M, Wang Y, Li Q, Chen F. A retrospective analysis of 38 carotid cavernous fistula patients treated with balloon-assisted endovascular fistula embolization through simultaneous transarterial and transvenous approaches. Int J Clin Exp Med 2016;9(10):19399-19407

20 Wang W, Li MH, Li YD, Gu BX, Lu HT. Reconstruction of the internal carotid artery after treatment of complex traumatic direct carotid-cavernous fistulas with the Willis covered stent: a retrospective study with long-term follow-up. Neurosurgery 2016;79(6):794-805

21 Wendl CM, Henkes H, Martinez Moreno R, Ganslandt O, Bäzner H, Aguilar Pérez M. Direct carotid cavernous sinus fistulae: vessel reconstruction using flow-diverting implants. Clin Neuroradiol 2017;27(4):493-501 